Check for updates

Cite this: RSC Adv., 2019, 9, 30519

\title{
The effect of proton irradiation on the properties of a graphene oxide paper
}

\author{
Xiaojuan Zhen, (D) ${ }^{\mathrm{b}}$ Yifan Huang, $\dot{t}^{* a}$ Shengsheng Yang, $\S^{\star \mathrm{bd}}$ Zhanzu Feng, ${ }^{\mathrm{d}}$ \\ Dedong $\mathrm{Ba}^{\mathrm{bc}}{ }^{\mathrm{b}}$ Jianhong Zhuang, ${ }^{\mathrm{bc}} \mathrm{Yi}_{\mathrm{i}}$ Wang $^{\mathrm{b}}$ and Xiaogang Qin ${ }^{\mathrm{bc}}$
}

A graphene oxide paper (GOP) was irradiated with $500 \mathrm{keV}$ proton for total fluence of $2 \times 10^{13} \mathrm{~cm}^{-2}$ to $2 \times$ $10^{15} \mathrm{~cm}^{-2}$ in a ground-based irradiation simulator. The spacing of layer, surface chemical composition, structural defects, thermal conductivity and electrical property of the GOP before and after irradiation was measured. The results indicated that the spacing of layer decreased after irradiation. The ratio of total carbon atom and total oxygen atom increased from 2.40 to 4.31 as well as the $\mathrm{sp}^{2}$ hybridized carbons obviously increased after $2 \times 10^{15} \mathrm{~cm}^{-2}$ irradiation. The XPS analysis suggested the occurrence of reduction, and the Raman spectra indicated that the defects were produced after proton irradiation. Furthermore, the thermal conductivity of GOP decreased, and then increased smoothly as the irradiation fluences were increased, and the electrical property showed the similar trend. The change in the thermal and electrical properties for GOP could be attributed to the defects and the removal of oxygencontaining functional groups, which lead to the phonon conduct path and scattering centers changed under proton irradiation. This study could promote the application of GOP in future space expeditions.

Received 14th July 2019

Accepted 19th August 2019

DOI: 10.1039/c9ra05389a

rsc.li/rsc-advances spacecraft. ${ }^{3}$ Zhang et al. has demonstrated that graphene can be used as protection coatings under atomic oxygen erosion. ${ }^{4,5}$ The study of ultraviolet exposure effects on graphene and its field effect transistors are important keys on the design of electronic and optoelectronic devices for space missions. Emilie J. Siochi pointed out that the graphene used in aerospace mainly depends on reliable functionality like any other new material, ${ }^{3}$ so it is necessary to study the irradiation effect, such as gamma ray, energetic particles from space environment before they will be used in aeronautics and space applications.

The existing irradiation effect research on graphene is mainly focused on materials preparation and property modification, and the experiment materials used are small-sized or thin films. For instance, some studies show that the gamma ray irradiation is an effective and green approach for the reduction, functionalization, the synthesis of nanocomposites and the selfassembly of oxide graphene sheets. ${ }^{6}$ The majority of research for energetic particles, including ion and electron effects on graphene, includes modification in property and structure..$^{7-12}$ Chen et al. found that graphene oxide (GO) can be partially reduced by electron beam irradiation, ${ }^{12}$ and the adsorptive performance of the reduced graphene oxide (rGO) also can be improved. ${ }^{13}$ The research indicates that ion irradiation is a useful way for the modification of GO and also for tailoring its structure, ${ }^{14-16}$ and the electron emission properties can be enhanced by irradiation. ${ }^{\mathbf{1 4}}$ Tyagi et al. has exhibited in their published study that the maximum lattice temperature of a GO film (GOF) can be changed by $\mathrm{C}$ ion irradiation. ${ }^{17}$ The changes brought in structure and property was defects, orders of 
magnitude and amorphization induced by irradiation. These researches have an undeniable role in promoting the space application of graphene, though the initial intention was not based on this aim. There are also some studies based on the space radiation effect on graphene materials, in which the materials in the experiments are usually suspended graphene, with size as the limitation. .118 $^{\mathbf{1}}$

The charged particles from near-Earth space and solar cosmic rays, including high-speed protons, are a threat to astronauts and satellites. The most damaging effects are known to be the charging of the surfaces and electrostatic discharge in the spacecraft. The study of proton irradiation damage in a mono-layer graphene suggests that the energy/dose has a direct relationship on defect formation. ${ }^{18}$ The research by Chen et al. shows that the structural defects and electrical characteristics of graphene transistors change after different energy proton irradiations. ${ }^{19}$ Mathew et al. have also found that the stability and the layer number of graphene on a $\mathrm{SiO}_{2} / \mathrm{Si}$ substrate fabricated by micro-mechanical exfoliation are influenced by focused proton irradiation. ${ }^{20}$ Therefore, there is lack of irradiation effect on the bulk graphene materials synthesized by a chemical method, which is considered to be a promising method for mass production. For this, the proton effect on bulk GO materials has been studied in this paper.

A free standing GO paper (GOP) is a fascinating material possessing excellent mechanical properties and chemical stability, and its potential applications cover isotropic ionic conductors, thermal conductive films, mechanically reinforced composites or transparent materials. ${ }^{\mathbf{9 , 1 0 , 1 5 , 2 1}}$ In the AP- $8_{\max }$ trapped proton environment of the Earth's radiation belt, the proton irradiation energy is in the range of $0.1-400 \mathrm{MeV} .^{22}$ The current research focussed on the 5-20 MeV proton irradiation on a monolayer or a thin film graphene, ${ }^{\mathbf{1 8 , 2 3 , 2 4}}$ and Korkut et al. have studied the $500 \mathrm{keV}$ proton irradiation on supported bilayer graphene by a simulation method. ${ }^{25}$ In this study, we have adopted the AP- $8_{\text {max }}$ mode to discuss the $500 \mathrm{keV}$ proton effect irradiation on bulk GOP. The total purpose is to promote the bulk graphene in harsh space applications.

\section{Experimental}

\subsection{GOP preparation}

GOP was purchased from JCNANO company in China with the thickness of about $50 \pm 10 \mu \mathrm{m}$, size $6 \mathrm{~cm} \times 6 \mathrm{~cm}$ and the density of $1.30 \mathrm{~g} \mathrm{~cm}^{-3}$, respectively, which was obtained by the Hummers' method. The materials were vacuumed for $24 \mathrm{~h}$, which were maintained at $0.1 \mathrm{~Pa}$ to prevent contamination and ensure that the desorption processes finished.

\subsection{Proton irradiation experiment}

The proton irradiation experiment was performed using a ground-based irradiation simulator at Lanzhou Institute of Physics (LIP), China Academy of Space Technology (CAST) 730000. (CAST, Lanzhou, China). The irradiation of the samples was conducted by $500 \mathrm{keV}$ proton with the flux of $1 \times 10^{10} \mathrm{~cm}^{-2}$ $\mathrm{s}^{-1}$ for ion fluences $2 \times 10^{13}, 2 \times 10^{14}$ and $2 \times 10^{15} \mathrm{~cm}^{-2}$. The pressure was kept in vacuum of $10^{-5} \mathrm{~Pa}$ at room temperature in an irradiation chamber.

\subsection{Material characterization techniques}

To study the proton irradiation on GOP, the X-ray diffraction (XRD) were conducted using $\mathrm{Cu} \mathrm{K} \alpha$ radiation $(\lambda=0.154 \mathrm{~nm})$ with a rate of $5^{\circ} \mathrm{min}^{-1}$ at room temperature. The X-ray photoelectron spectroscopy (XPS, EscaLab $250 \mathrm{Xi}$ spectrometer) was performed to determine the chemical state of the GOP. The laser Raman spectroscopy was performed using an HR Raman spectrometer $532 \mathrm{~nm}$ laser with $\mathrm{Nd}$ filter $0.1 \%$ to get the information about the structure composition. The thermal diffusion coefficient of GOP was measured using a thermal conductivity meter (LFA laser flash apparatus, Netzsch LFA 467) in plane. The fracture surface of a pristine GOP was observed using a field emission scanning electron microscope (FE-SEM, Hitachi S4800). The electrical property of GOP was investigated by square resistance test with a four-point probe (RTS-9 KEITHLEY 2400). The XRD and XPS experiments were performed at Lanzhou university, and the Raman spectroscopy and SEM tests were performed at Lanzhou Institute of Chemical Physics, Chinese Academy of Sciences, and the thermal conductivity test were performed at NETZSCH Scientific Instruments Trading (Shanghai, China), respectively. The test results of GOP before and after proton irradiation were evaluated and compared.

\section{Results and discussion}

\subsection{X-ray diffraction (XRD) analysis}

The XRD patterns of GOP before and after irradiation are shown in the inset of Fig. 1. It can be seen that the XRD patterns of the pristine GOP display a sharp and intense peak located at $9.10^{\circ}$, and the corresponding $d$-spacing (the distance of layer-to-layer) is $0.48 \mathrm{~nm}$ according to the Scherrer's equation as show in expression (1). The diffraction peak gradually shifts to $10.14^{\circ}$ with the $d$-spacing $0.44 \mathrm{~nm}$ after proton irradiation with the fluences $2.0 \times 10^{13}$ and $2.0 \times 10^{15} \mathrm{~cm}^{-2}$. The intensity of the domain peak decreased dramatically, which may be due to the removal of oxygen-containing functional groups accompanied with the edges and basal plane of materials, and the increase in broadness of diffraction peaks with irradiation fluences indicates a disordered structure..$^{7,12,26,27}$

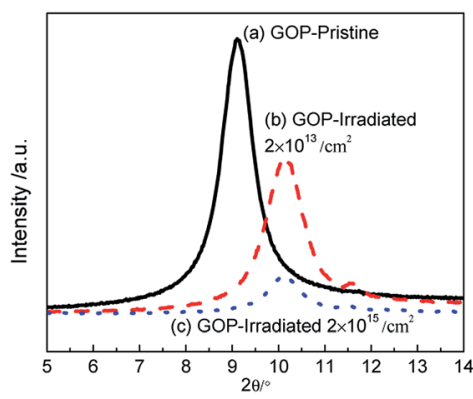

Fig. 1 The XRD patterns of pristine and irradiated GOPs. 


$$
d=\frac{n \lambda}{2 \sin \theta}
$$

where $d$ is the layer-to-layer distance, $n$ is the order, $\lambda$ is the wavelength of X-ray and $\theta$ is the corresponding angle of XRD peak.

\subsection{X-ray photoelectron spectroscopy (XPS) analysis}

To further study the proton effect on GOP, the changes in the functional compositions are analyzed by XPS, and the $\mathrm{C}$ 1s peaks are divided into different curves according to symmetric Gaussian. Fig. 2 is the $\mathrm{C}$ 1s peaks of the pristine and GOP after irradiation, respectively, and the results are presented in Table 1. The GOP before and after irradiation show five kinds of binding energies in Fig. 2(a)-(c). The dominant peak at $284.1 \mathrm{eV}$ is attributed to the existence of $\mathrm{sp}^{2}$ hybridized carbon $\left(\mathrm{C}=\mathrm{C}\right.$ bond) as well as $\mathrm{sp}^{3}$ hybridized carbons (C-C, $284.8 \mathrm{eV}$ ), and other peaks, including $\mathrm{C}-\mathrm{O}$ bond (hydroxyl and epoxy), $\mathrm{C}=\mathrm{O}$ bond (carbonyl) and $\mathrm{C}-\mathrm{OOH}$ bond (carboxyl) appears around $286.7 \mathrm{eV}, 287.7 \mathrm{eV}$ and $288.7 \mathrm{eV}$, respectively. A similar result has been reported for others. ${ }^{10}$ It can be seen from Fig. 2(b) and Table 1 that the $2 \times 10^{14} \mathrm{~cm}^{-2}$ irradiated GOP show a smooth change in functional compositions,
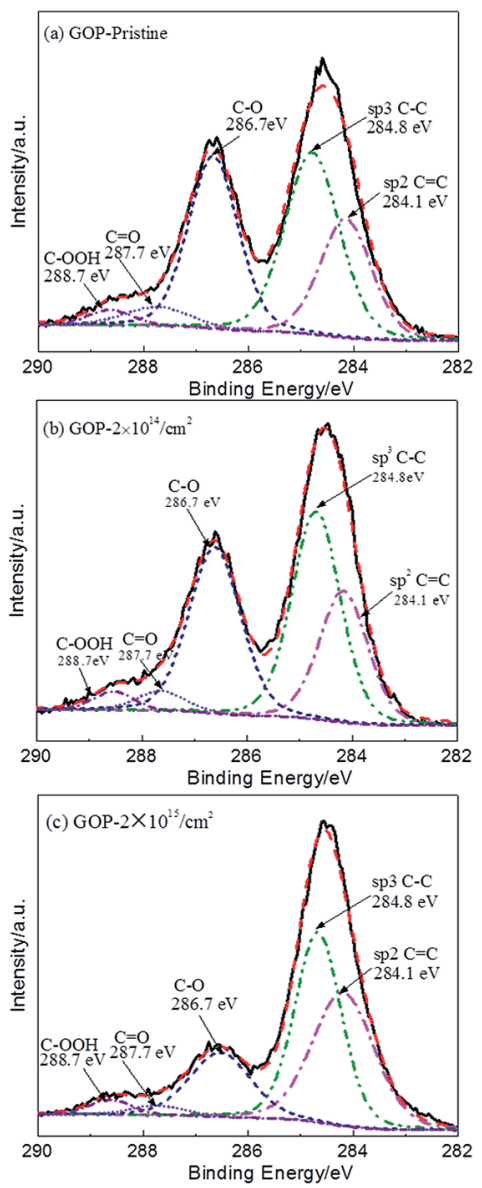

Fig. 2 XPS results of the pristine GOP (a) and the irradiated GOP with the fluences of (b) $2 \times 10^{14} \mathrm{~cm}^{-2}$ and (c) $2 \times 10^{15} \mathrm{~cm}^{-2}$ under the 500 $\mathrm{keV}$ proton irradiation, respectively.
Table 1 The results of XPS analysis for pristine and irradiated GOP

\begin{tabular}{|c|c|c|c|c|}
\hline \multirow[b]{2}{*}{ Groups } & \multirow{2}{*}{$\begin{array}{l}\text { Binding Energy } \\
(\mathrm{eV})\end{array}$} & Pristine & $\begin{array}{l}2 \times \\
10^{14} \mathrm{~cm}^{-2}\end{array}$ & $\begin{array}{l}2 \times \\
10^{15} \mathrm{~cm}^{-2}\end{array}$ \\
\hline & & \multicolumn{2}{|c|}{ Content (\%) } & \\
\hline $\mathrm{sp}^{2} \mathrm{C}=\mathrm{C}$ & 284.1 & 22.96 & 23.68 & 37.15 \\
\hline $\mathrm{sp}^{3} \mathrm{C}-\mathrm{C}$ & 284.8 & 37.46 & 37.55 & 39.79 \\
\hline $\mathrm{C}-\mathrm{O}$ & 286.7 & 32.35 & 32.27 & 17.76 \\
\hline $\mathrm{C}=\mathrm{O}$ & 287.7 & 4.66 & 3.63 & 2.40 \\
\hline $\mathrm{C}-\mathrm{OOH}$ & 288.7 & 2.57 & 2.87 & 2.90 \\
\hline $\mathrm{sp}^{3} / \mathrm{sp}^{2}$ & - & 1.63 & 1.59 & 1.07 \\
\hline $\mathrm{C} / \mathrm{O}$ & - & 2.30 & 2.77 & 4.31 \\
\hline
\end{tabular}

while the total carbon to total oxygen $(\mathrm{C} / \mathrm{O})$ atomic ratio increased to 2.77 . When the irradiation fluences increase to $2 \times 10^{15} \mathrm{~cm}^{-2}$, the content of oxygen-containing functional groups decreased from $39.58 \%$ for the pristine GOP to $23.06 \%$ for the irradiated $\mathrm{GOP}$, while value of $\mathrm{C} / \mathrm{O}$ atomic ratio was increased from 2.30 to 4.31. Moreover, the ratio of the $\mathrm{sp}^{3}$ hybridized atoms and the $\mathrm{sp}^{2}$ hybridized atoms was decreased by $34.35 \%$. The $\mathrm{C}-\mathrm{O}$ bond and the $\mathrm{C}=\mathrm{O}$ content was decreased appreciably, whereas the $\mathrm{COOH}$ bond content gradually increased. The decrease in hydroxyl, epoxide and carbonyl indicates the reduction action that happened in GO, ${ }^{6,28-31}$ whereas we speculated that the GOP was highly reduced under higher irradiation fluences. Meanwhile, it is suggested that the $\mathrm{C}-\mathrm{O}$ components was converted to $\mathrm{sp}^{2}$ hybridized carbon under energetic proton knocking on carbon atoms. Moreover, the $\mathrm{sp}^{2}$ hybridized carbon was easily formed than $\mathrm{sp}^{3}$ hybridized carbon under proton irradiation.

\subsection{Raman spectroscopy analysis}

Raman spectroscopy is used as a common tool for studying the structural composition of GOP, which is an invaluable method in graphene studies. ${ }^{\mathbf{1 0 , 2 9 , 3 1 - 3 6}}$ It can be used to confirm the vibrational modes of surface functional groups and structural

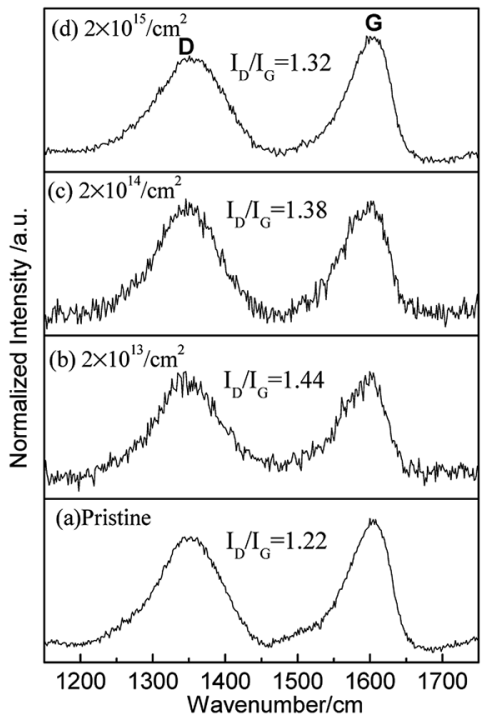

Fig. 3 Raman spectra of the pristine and irradiated GOP. 
damage. Fig. 3 shows the Raman spectra of GOP for the pristine and irradiated samples. Both of the spectra show a D peak positioned at $1345 \mathrm{~cm}^{-1}$ and a $\mathrm{G}$ peak positioned at $1590 \mathrm{~cm}^{-1}$. The $\mathrm{G}$ band indicates the origin of the stretching vibration of the $\mathrm{sp}^{2}$ carbon atoms at the Brillouin zone center and its existence is considered neglecting the order or disorder of $\mathrm{sp}^{2}$ carbons in the material. ${ }^{37-40}$ In a perfect graphite structure, the vibrational mode associated with $\mathrm{D}$ peak is not permitted and only appears for six-fold carbon rings located near structural defects. Therefore, the observation of $\mathrm{D}$ peak is a common method for the detection of defect formation in carbon materials, and the $\mathrm{D}$ band is representative of defects, such as bondangle disorder, bond-length disorder, vacancies, edge defects, amorphous, involving the transverse optical phonons near the $\kappa$-point phonons. Therefore, the ratio of the peak intensities $I_{\mathrm{D}} /$ $I_{\mathrm{G}}$ (D peaks and $\mathrm{G}$ peaks) is a typical analysis method to evaluate defect formation and the structure order in a qualitative way. The strong D peak refers to the defects produced during fabrication and the influence of structure edges in the pristine GOP. ${ }^{31}$ For pristine GOP irradiated with different fluences, the value $I_{\mathrm{D}} / I_{\mathrm{G}}$ was first increased from 1.22 to 1.44 and then decreased to 1.32. Furthermore, the FWHM of the D and $G$ peaks for pristine is $95 \mathrm{~cm}^{-1}$ and $66 \mathrm{~cm}^{-1}$ but then increases to $106 \mathrm{~cm}^{-1}$ and $70 \mathrm{~cm}^{-1}$ after the irradiation of $2 \times 10^{15} \mathrm{~cm}^{-2}$ fluences, respectively. The results indicate that the large numbers of defects are created in the lower $2 \times 10^{13} \mathrm{~cm}^{-2}$ and 2 $\times 10^{14} \mathrm{~cm}^{-2}$ fluences irradiated GOP. The D and G peaks are wide in higher $2 \times 10^{15} \mathrm{~cm}^{-2}$ fluence irradiated GOP, and it can be concluded that the disorder structure is formed, and the higher value $I_{\mathrm{D}} / I_{\mathrm{G}}$ suggest the $\mathrm{C}=\mathrm{C}$ bond ( $\mathrm{sp}^{2}$ carbon) distortion, and the non-six ring carbon rings production..$^{18,29,31,35,38}$

In general, the $I_{\mathrm{D}} / I_{\mathrm{G}}$ value is very sensitive to defects and sixring structure ${ }^{26}$ while the broadening of the FWHM of D and G peaks always represent a structure disorder. ${ }^{\mathbf{4 1 , 4 2}}$ Many previous studies suggest that there is a positive correlation relation between the number of defects and $I_{\mathrm{D}} / I_{\mathrm{G}}$ ratio in graphene. ${ }^{18,43} \mathrm{In}$ this study, the $I_{\mathrm{D}} / I_{\mathrm{G}}$ value increases obviously after $2 \times 10^{13} \mathrm{~cm}^{-2}$ irradiation fluences in GOP, which indicates that the proton irradiation lead to a high defect density. In the $2 \times 10^{15} \mathrm{~cm}^{-2}$ fluence irradiated GOP, the decline in the $I_{\mathrm{D}} / I_{\mathrm{G}}$ value suggests the lower defect density compared to lower fluence irradiated materials, which is attributed to the complete removal of oxygencontaining functional groups. Combined with the above XRD and XPS analysis, the findings indicate that although the amount of oxygen-containing functional groups is reduced compared to that in pristine GOP, many defects are retained after the high fluence irradiation process. ${ }^{10,18,23,29,32-36,41,43}$

\subsection{Thermal conductivities}

Recently the thermal properties of lower dimensional graphene have attracted considerable interest among scientific and engineering communities. The heat transport is a key factor for the graphene in the electronic industry. Therefore, the thermal performance of GOP has been studied in this study. The thermal diffusivity in plane of the sample can be directly obtained by LFT transient method. The circle shaped sample with the diameter of $2.5 \mathrm{~mm}$ is heated from one side, and then the temperature change is obtained on the opposite side. The thermal conductivity is obtained according to the eqn (2).

$$
k=\alpha C_{\mathrm{p}} \rho
$$

where $k \mathrm{~W}(\mathrm{~m} \mathrm{~K})^{-1}$ is the thermal conductivity that needs to be calculated, $\alpha$ is the thermal diffusivity, $C_{\mathrm{p}} \mathrm{J}(\mathrm{g} \mathrm{K})^{-1}$ is the specific heat capacity assumed as value $0.708,{ }^{42}$ and $\rho\left(\mathrm{g} \mathrm{cm}^{-3}\right)$ denotes the volume density of the samples. In order to get the $\rho$, weight of the GOP is measured in a microbalance, and the thickness is measured by SEM. The average value of the thickness of GOP is obtained from the cross-section structure that can be observed in Fig. 4. From the microstructure of the cross-section, it can be found that the materials have layered structure.

Table 2 shows the thermal diffusivity and thermal conductivity in plane. It can be seen that the thermal conductivities of pristine GOP is $2.795 \mathrm{~W}(\mathrm{~m} \mathrm{~K})^{-1}$, which may be caused by the lower quality of a layer structure and due to the influence of the defects. The thermal property of irradiated GOP shows that the thermal conductivity is decreased mildly and then increased to $2.982 \mathrm{~W}\left(\mathrm{~m} \mathrm{~K}^{-1}\right.$ with the irradiation fluences of up to $2 \times 10^{15}$ $\mathrm{cm}^{-2}$. The thermal transport in carbon materials is mainly determined by acoustic phonons, and the phonon can be further influenced by the defects and structure disorder. ${ }^{44-49}$ The defects, disorder structure and the oxygen functional group are influenced by proton irradiation, leading to the change in the thermal properties of GOP.

\subsection{Electrical property}

Now the study of electrical properties for graphene is emphasized on reinforcing the conductivity of polymers, and there are limited studies on pure bulk materials, particularly GO. Here, we have tested the electrical resistance of GOP before and after proton irradiation, as show in Fig. 5. The square resistance of the pristine GOP is $4.08 \mathrm{M} \Omega$ and $83.18,76.92$ and $6.28 \mathrm{M} \Omega$ after $2 \times 10^{13}, 2 \times 10^{14}$ and $2 \times 10^{15} \mathrm{~cm}^{-2}$, respectively. The resistance of GOP materials show remarkable increase and then decrease, while the value also exceeds that of pristine $53.9 \%$ after irradiation fluences are made to the highest, similar to thermal conductivity. It is demonstrated that the proton irradiation effect caused the changes in electrical conductivity. The increment in the square resistance after irradiation can be explained by the number of defects. A point defect is the primary effect on electrical conduction degradation in

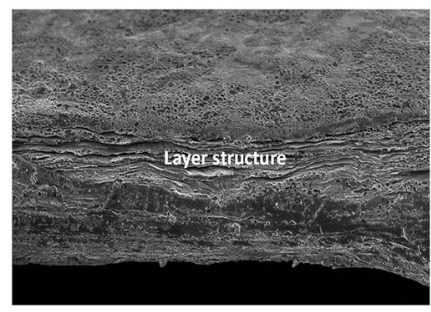

Fig. 4 SEM images of pristine GOP from the cross-sectional view. 
Table 2 The thermal conductivity results of GOP before and after proton irradiation

\begin{tabular}{|c|c|c|c|}
\hline Sample & Irradiation fluences & Thermal diffusion & $\begin{array}{l}\text { Thermal } \\
\text { conductivity }\end{array}$ \\
\hline Pristine & 0 & $3.058 \mathrm{~m}^{2} \mathrm{~s}^{-1}$ & $2.795 \mathrm{~W} \mathrm{~m}^{-1} \mathrm{~K}^{-1}$ \\
\hline Irradiated & $2 \times 10^{13} \mathrm{~cm}^{-2}$ & $2.980 \mathrm{~m}^{2} \mathrm{~s}^{-1}$ & $2.723 \mathrm{~W} \mathrm{~m}^{-1} \mathrm{~K}^{-1}$ \\
\hline Irradiated & $2 \times 10^{14} \mathrm{~cm}^{-2}$ & $2.906 \mathrm{~m}^{2} \mathrm{~s}^{-1}$ & $2.660 \mathrm{~W} \mathrm{~m}{ }^{-1} \mathrm{~K}^{-1}$ \\
\hline Irradiated & $2 \times 10^{15} \mathrm{~cm}^{-2}$ & $3.263 \mathrm{~m}^{2} \mathrm{~s}^{-1}$ & $2.982 \mathrm{~W} \mathrm{~m}^{-1} \mathrm{~K}^{-1}$ \\
\hline
\end{tabular}

graphene, and the defects increase as well as cause scattering of the charge carriers. ${ }^{7,10,19,49-52}$ The resistance increase in the initial stage may be due to the high defect density, and the decrease may be related to the removal of oxygen-containing functional groups.

\subsection{Discussion}

For the above analysis, the proton irradiation effect on GOP shows the change of structure and property. The XRD results represent a small decrease of $d$-spacing for irradiated GOP with highest fluences $2 \times 10^{15} \mathrm{~cm}^{-2}$, which is possibly put down to the removal of oxygen-containing functional groups in a significant reduction eventually. ${ }^{11,53-55}$ The presence of a more disordered structure imputes the damage after high fluence proton irradiation. The surface compositions of the GOP show the reduction action event under proton irradiation. It can be explained by the oxygen-containing functional groups decrease for the highest fluence irradiated samples, while the $\mathrm{sp}^{2}$ hybridized carbon increase remarkably. The hydroxyl, epoxy and carbonyl groups may be more easily turned to $\mathrm{sp}^{2}$ carbon because of better electronic transfers under reduction process induced by proton irradiation. The peak area ratio of $I_{\mathrm{D}} / I_{\mathrm{G}}$ for 2 $\times 10^{13} \mathrm{~cm}^{-2}$ fluence irradiated GOP increases significantly compared to that of pristine, which indicates the defects introduced under energetic proton irradiation. The ion irradiation can induce polymorphic atomic defects in graphene, ${ }^{56,57}$ which generally contain vacancies, adatom-vacancy, and reconstructed..$^{18,23,24}$ The possible models of vacancies involve the formation of single vacancies (SVs) and double vacancies (DVs), and the adatom-vacancy pair models are constituted by vacancy and adatom, and reconstructions are by Stone-Wales (SW) that take place due to rotating or breaking and the recombination of the $\mathrm{C}=\mathrm{C}$ bonds. ${ }^{18}$ The energetic ion irradiation damage effect caused on graphene is due to the displacement and excitation of atoms..$^{29,31,33,34,56,57}$ In this experiment, when the $500 \mathrm{keV}$ proton hit against the surface of GOP, the energy of the colliding particles could transform to the target lattice carbon atoms, which caused the carbon atoms to deviate from the initial position, and the defects are formed when these atoms are knocked away. Under the lower $2 \times 10^{13} \mathrm{~cm}^{-2}$ irradiation fluences, the defects may originate from the vacancy modal in GOP. In the higher fluence irradiated samples, the XPS results indicate the creation of a $\mathrm{sp}^{2}$ carbon, which originate from the strong removal of the oxygen-containing functional group through the bond rupture. It can be conjectural that the defects came from the non-six carbon ring. Therefore, it can be concluded that the proton irradiation effect on GOP are defects formation, reduction and structure modification.

In this study, the thermal property of the pristine GOP shows a lower thermal conductivity, which may be caused by the low content of $\mathrm{sp}^{2}$ carbon and lots of defects. The heat transport in all bulk carbon allotropes is mostly carried by acoustic phonons, which are attributed to the strong covalent $\mathrm{sp}^{2}$ bonding, leading to efficient heat transfer with lattice vibrations. ${ }^{44-48}$ Moreover, the impurities and defects are the powerful primary factors that affect the phonon scatterings in graphene. ${ }^{49}$ We consider that the small thermal conductivity in GOP before and after lower fluence irradiation is attributed to defects and lesser amounts of $\mathrm{sp}^{2}$ carbons, respectively. When the irradiation fluences up to $2 \times 10^{15} \mathrm{~cm}^{-2}$ are used, the slight increase in the thermal conductivity may be induced by two factors. First, the irradiation introduces a higher $\mathrm{sp}^{2}$ carbon content, which can improve the efficiency of scattering for phonon transport. Second, the thermal conductivity slightly increases may be due to the removal of large number of oxygencontaining functional groups. The study suggests that a well interface can reduce the interfacial thermal, and good orientation can provide energetic pathways for phonon conduction. ${ }^{42}$ From this point, the removal of oxygen-containing functional groups may be the key factor to improve the quality of interface. The ion irradiation have limited the depth of penetration in graphene, ${ }^{25,29,31}$ and we have calculated the stopped range of 500 $\mathrm{keV}$ protons in GOP to be about $8.29 \mu \mathrm{m}$, so the change in defects and chemical composition may not obviously be attributed to the increase in depth, which can give a reasonable explanation for thermal properties before and after irradiation GOP. Furthermore, the GOP irradiated with $2 \times 10^{13} \mathrm{~cm}^{-2}$ exhibits the highest electrical resistance, which is correlated with plenty of oxygen functional groups remaining in the material and the increase in defects. The oxygen-containing

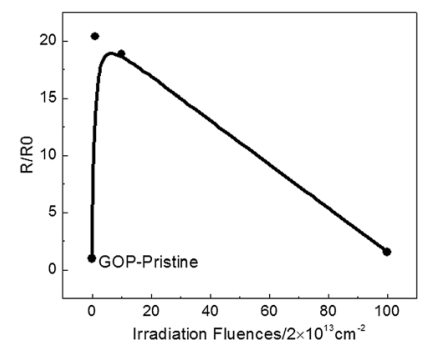

Fig. 5 The resistance result of pristine and irradiated GOP. 
functional groups are strong scattering centers, which can affect the electrical transport in graphene, and the defect density also can impact the scattering of charge carriers. ${ }^{10,50-52}$ When the irradiation fluences are increased to the highest $2 \times 10^{15} \mathrm{~cm}^{-2}$, its more complete reduction action led to the increase in content of $\mathrm{sp}^{2}$ and $\mathrm{sp}^{3}$ carbon, still retaining many defects, so the resistance is higher than that of pristine GOP but lower than other irradiated ones.

\section{Conclusion}

We have demonstrated the $500 \mathrm{keV}$ proton irradiation effect on GOP produced by Hummers' method. The irradiation brought about the reduction process and the defects. The analysis of XRD indicates the small $d$-spacing caused by relatively complete reduction in GOP after the highest fluences of $2 \times 10^{15} \mathrm{~cm}^{-2}$. The chemical compositions on the surface of the materials show a decrease in the oxygen-containing functional group and remarkable increase in the $\mathrm{sp}^{2}$ carbon, which further confirms the induced reduction by proton irradiation under higher fluence irradiation. The increase in the $I_{\mathrm{D}} / I_{\mathrm{G}}$ value indicates that a large number of defects are formed under lower $2 \times 10^{13} \mathrm{~cm}^{-2}$ irradiation fluences, which may be caused by the carbon atoms displacement under collisions of proton. In summary, the defect formation and the reduction action are the main effects in proton effect process. The irradiation effect on thermal conductivity and electrical properties is related to the number of defects, the $\mathrm{sp}^{2}$ carbon and oxygen-containing functional group content. The study illustrate that these bulk GO materials can be used as thermal or electrical conductivity coating, or electronic devices in future space missions.

\section{Conflicts of interest}

There are no conflicts to declare.

\section{Acknowledgements}

The work is supported by the National Natural Science Foundation of China (No. 11605079), and Fundamental Research Funds for the Central Universities (lzujbky-2019-kb06), and Open Fund of National Key Laboratory of Materials Behavior and Evaluation Technology in Space Environment (ZZYY-KFJJLXTZ03). Thanks for the help of Qiqi Yang with the measurements.

\section{References}

1 K. S. Novoselov, V. I. Fal ko and L. Colombo, Nature, 2012, 490, 192-200.

2 X. Wu, F. W. Mu and Y. H. Wang, Comput. Mater. Sci., 2018, 149, 98-106.

3 E. J. Siochi, Nat. Nanotechnol., 2014, 9, 745-747.

4 H. J. Zhang, S. M. Ren and J. B. Pu, Appl. Surf. Sci., 2018, 444, 28-35.

5 W. Zhang, M. Yi and Z. Shen, J. Mater. Sci., 2013, 48, 24162423.
6 L. F. Dumée, C. F. Feng and L. He, Appl. Surf. Sci., 2014, 322, 126-135.

7 C. Tyagi, G. B. V. S. Lakshmi and S. Kumar, Nucl. Instrum. Methods Phys. Res., Sect. B, 2016, 379, 171-175.

8 K. H. Wu, H. H. Cheng and A. A. Mohammad, Carbon, 2015, 95, 738-745.

9 S. Gupta, E. Heintzmana and J. Jasinskib, J. Raman Spectrosc., 2015, 46, 509-523.

10 O. O. Voitsihovska, R. M. Rudenko and V. Y. Povarchuk, Mater. Lett., 2019, 236, 334-336.

11 G. Gawlik, P. Ciepielewski and J. M. Baranowski, Surf. Coat. Technol., 2016, 306, 119-122.

12 L. Chen, Z. W. Xu and J. L. Li, Mater. Lett., 2011, 65, 12291230.

13 P. Liu, W. Qi and W. Z. An, Nano, 2015, 10, 3.

14 H. Sharma, D. C. Agarwal and M. Sharma, J. Phys. D: Appl. Phys., 2013, 46, 315301.

15 P. Malinský, A. Macková, R. Mikšová, H. Kováčiková, M. Cutroneo, J. Luxa, et al., Phys. Chem. Chem. Phys., 2017, 19(16), 10282-10291.

16 A. Olejniczak, N. A. Nebogatikova and A. V. Frolov, Carbon, 2019, 141, 390-399.

17 T. Chetna, S. A. Khan, S. Ojha, D. K. Avasthi and A. Tripathi, Vacuum, 2018, 154, 259-263.

18 G. S. Yang, B.-J. Kim and K. Kim, RSC Adv., 2015, 5, 31861.

19 Y. Chen, H. Y. Zhao and Y. Y. W. Radiat, Phys. Chem., 2018, 153, 79-85.

20 S. Mathew, T. K. Chan and D. Zhan, J. Appl. Phys., 2011, 110(8), 084309.

21 C. Teng, D. Xie and J. F. Wang, Adv. Funct. Mater., 2017, 27, 1700240.

22 D. M. Sawyer and J. I. Vette, Nasa Sti/recon Technical Report 77, (1976).

23 S. Lee, J. Seo, J. Hong, et al., Appl. Surf. Sci., 2015, 344, 52-56.

24 J. Y. Kim, Y. H. Go, J. Y. An, H. Cho, S. Oh and Y. H. Kahng, Mater. Res. Express, 2018, 6, 015605.

25 H. Korkut and T. Korkut, J. Radioanal. Nucl. Chem., 2014, 299, 13-17.

26 S. M. Clark, K.-J. Jeon and J.-Y. Chen, Solid State Commun., 2013, 154, 15-18.

27 M. Enayati, A. Nemati, A. Zarrabi and M. A. Shokrgozar, J. Alloys Compd., 2019, 784, 134-148.

28 K. S. Andrikopoulos and G. Bounos, Adv. Mater. Interfaces, 2014, 1(1-10), 1400250.

29 P. Malinský, M. Cutroneo, A. Macková, V. Hnatowicz, K. Szökölová and M. Bohačová, Surf. Interface. Anal., 2018, 50(1), 1-6.

30 A. Heya and N. Matsuo, International Workshop on Activematrix Flatpanel Displays \& Devices, 2016.

31 P. Kumar, B. Das, B. Chitara, K. Subrahmanyam, K. Gopalakrishnan, S. B. Krupanidhi and C. N. R. Rao, Macromol. Chem. Phys., 2012, 213(10-11), 1146-1163.

32 P. Malinsky, A. Macková, M. Florianová, M. Cutroneo, et al., Phys. Status Solidi B, 2019, 256, 1800409.

33 A. C. Ferrari, J. C. Meyer, V. Scardaci, C. Csiraghi, M. Lazzeri and F. Mauri, Phys. Rev. Lett., 2006, 97(18), 187401. 
34 L. Chen, Z. Xu, J. Li, C. Min, L. Liu, X. Song, et al., Mater. Lett., 2011, 65(8), 1229-1230.

35 J. D. Renteria, S. Ramirez, H. Malekpour, B. Alonso, A. Centeno, A. Zurutuza, A. I. Cocemasov, D. L. Nika and A. A. Balandin, Adv. Funct. Mater., 2015, 25(29), 4664-4672. 36 C. Coleman, R. Erasmus and S. Bhattacharyya, Appl. Phys. Lett., 2016, 109(25), 253102.

37 L. Liu, Z. G. Shen, S. S. Liang, et al., J. Appl. Poylm. Sci., 2014, 131(11), 2928-2935.

38 Q. Zhao, S. J. Gang and J. K. Min, Sci. Adv., 2017, 3, e1601536.

39 B. Lanfant, Y. Leconte and N. Debski, Ceram. Int., 2019, 45, 2566-2575.

40 M. Bruna, A. K. Ott and M. Ijas, ACS Nano, 2014, 8, 74327441.

41 A. A. Anthea, V. Andrea, C. Pascal, B. M. K. org and C. Glenn, Diamond Relat. Mater., 2018, 89, 163-173.

42 A. C. Ferrari and D. M. Basko, Nat. Nanotechnol., 2013, 8(4), 235-246.

43 L. G. Canc ado, A. Jorio, E. H. Martins Ferreira and F. Stavale, Nano Lett., 2011, 11, 3190-3196.

44 D. Estrada, Z. Y. Li, G. M. Choi, S. N. Dunham, A. Serov, J. Lee, Y. F. Meng, F. F. Lian, N. C. Wang, et al., npj $2 D$ Mater. Appl., 2019, 10.
45 Y. F. Zhang, J. Shi and C. Chen, Phys. E, 2018, 97, 151-154. 46 W. Yu, H. Q. Xie and F. X. Li, Appl. Phys. Lett., 2013, 103, 141913.

47 J. D. Renteria, S. Ramirez and H. Malekpou, Adv. Funct. Mater., 2015, 25, 4664-4672.

48 A. A. Balandin, Nat. Mater., 2011, 10, 569-581.

49 H. Guo, X. Li and B. A. Li, Mater. Des., 2017, 114, 355-363.

50 A. Alofi and G. P. Srivastava, J. Appl. Phys., 2012, 112, 013517.

51 S. Kumar, A. Kumar and A. Tripathi, J. Appl. Phys., 2018, 123, 161533.

52 J. A. Rodriguezmanzo, A. Balan, C. Nayor, W. Parkin, M. Puster and A. T. C. Johnson, et al., Aps March Meeting, (2015).

53 G. Jayalakshmi, K. Saravanan and B. K. Panigrahi, Nat. Nanotechnol., 2018, 29, 185701.

54 E. Abbe, T. Schüler and S. Klosz, J. Nucl. Mater., 2017, 495, 299-305.

55 Y. L. He, J. H. Li and L. F. Li, Mater. Lett., 2016, 177, 76-79.

56 L. P. Wang, X. Q. Fan, L. Wen, H. Li and M. Zhu, Nanoscale, 2017, 9(35), 13079-13088.

57 X. Q. Fan and L. P. Wang, Sci. Rep., 2015, 5, 12734. 